\title{
Sistema de información de mercado para el sector de agroalimentos de los mercados de la Provincia de Tungurahua.
}

Market information system for the agrifood sector of the markets of the Province of Tungurahua

Velasteguí López Efraín. ${ }^{1}$, Carrasco Ruano Tatiana. ${ }^{2}$ \& Wilma Mónica Chávez Chifla. ${ }^{3}$

DOI: https://doi.org/10.33262/visionariodigital.v2i1.33

\section{Resumen.}

La comercialización de agroalimentos en la provincia de Tungurahua es el principal mercado comercial del país, pues su mercado mayorista acoge a comerciantes y productores de todo el Ecuador, mismos que expenden ahí sus productos, por eso el desarrollo de este comercio es importante puesto que los precios de los productos a nivel nacional están determinados por los precios de comercialización que se den en la provincia de Tungurahua, sin olvidar que la variación de precios afecta a la economía nacional y sobre todo al bolsillo de los consumidores. Este proyecto estableció los sistemas de información utilizados actualmente cuyos objetivos son proporcionar información a las unidades de comercialización para mantener a los productores y comerciantes informados acerca de los precios vigentes y sus fluctuaciones relacionadas con productos agroalimenticios, y apoyar a los procesos de comercialización de los productores y comerciantes. La aplicación de esta propuesta dio paso a incrementar la comercialización de los productos agroalimenticios, en los mercados de la provincia de Tungurahua, por medio de información para realizar pronósticos apropiados de la futura conducta de productos para la siguiente feria, en función de la época, además tuvieron información de los costos de movilización de productos hasta los diferentes mercados.

Palabras clave: sistemas, comercialización, información, productos, mercado.

\footnotetext{
${ }^{1}$ Universidad Técnica de Ambato, Ambato, Ecuador, le.velastegui@uta.edu.ec

2 Universidad Internacional de La Rioja UNIR, España, tcarrascor@yahoo.cpm

${ }^{3}$ Universidad Técnica de Ambato, Ambato, Ecuador, wmony86@hotmail.com 


\begin{abstract}
.
The commercialization of agrofoods in the province of Tungurahua is the main commercial market of the country, because its wholesale market welcomes merchants and producers from all over Ecuador, who sell their products there, so the development of this trade is important since the prices of products at the national level are determined by the marketing prices in the province of Tungurahua, without forgetting that the price variation affects the national economy and especially the consumer's pocket. This project established the information systems currently used whose objectives are to provide information to marketing units to keep producers and traders informed about current prices and their fluctuations related to agro-food products and to support the marketing processes of producers and merchants. The application of this proposal gave way to increase the commercialization of agro-food products in the markets of the province of Tungurahua, by means of information to make appropriate forecasts of the future conduct of products for the following fair, depending on the time, in addition they had information of the costs of mobilization of products to the different markets.
\end{abstract}

KEYWORDS: Systems, Marketing, Information, Products, Market.

\title{
I. Introducción.
}

La alimentación es parte muy importante para el desarrollo de la vida humana, por lo tanto esta "constituye uno de los componentes esenciales del bienestar y un valioso indicador de los niveles de vida de una comunidad, y representa, junto con otros indicadores, el grado de desarrollo de un país. Una de las formas de expresión del atraso y la pobreza de una colectividad está dado por el hambre y la desnutrición" (Collazos, 2016, pág. 1), por lo tanto el acceso a alimentos de calidad está determinado por la producción y comercialización de productos de calidad y por supuesto de los precios de los mismos, de tal manera que los comerciantes y expendedores de agroalimentos deben siempre estar informados acerca de los temas que atañen a la producción y comercialización de los mismos, basado en esta consideración nace la necesidad de esta investigación, la misma que pretende determinar los beneficios de la implementación de un sistema de información apropiado, en la comercialización y producción de este tipo de alimentos, El alcance de los objetivos de esta investigación fue necesaria en primer lugar puesto que se pudo determinar que en los mercados de la provincia de Tungurahua existe un factor común en cuanto a la organización de sus comerciantes, este está determinado en que existen diferencias entre los comerciantes ya que es muy difícil que es estos encuentren el acuerdo para poder desarrollar sus actividades de comercialización de tal forma que es necesario que se les brinde una alternativa de solución a este inconveniente por medio de un sistema de información el cual aportará benéficamente al sector de agroalimentos ya que "Para minimizar las consecuencias es necesario realizar una serie de actuaciones o actividades que permitan minimizar el impacto negativo e incertidumbre que se genera por un cambio que se va a provocar en la organización” (García, Gonzalez, Rivera, \& Rivas, 2015). 
De esta manera la presente investigación que beneficiará al sector de agroalimentos de la provincia de Tungurahua hace que surja la siguiente interrogante ¿Cómo los sistemas de información aportarán al sector de agroalimentos de la provincia de Tungurahua?; pregunta que hace pensar en "una conciencia creciente acerca de la importancia que tienen las tecnologías de la información y la comunicación como herramientas para el logro de las metas del desarrollo económico y social, ya que en la actualidad se reconoce que es necesario lograr un adecuado balance entre la tecnología, las políticas públicas, la asignación de recursos y el establecimiento de metas concretas para alcanzar un impacto positivo en las actividades de comercialización" (Llanusa, Rojo, Caraballoso, Roberto, \& Pérez, 2015), de este modo la respuesta a esta pregunta se obtuvo en base a la investigación para poder establecer los medios y procesos apropiados para la implementación de un sistema de información por medio del cual se socializar de manera oportuna la información necesaria para que los comerciantes y productores puedan comercializar su productos sin tener que afrontar ciegamente las fluctuaciones de precios en el mercado, además podrán conocer los tipos de estrategias que manejan actualmente para dar a conocer los servicios y productos que se expenden al interior de los mercados de la provincia de Tungurahua, cabe recalcar que para esta investigación se contó con todo el respaldo del ente regulador y encargado de la Red de Plazas y Mercados Municipales de la provincia de Tungurahua,

Caber recalcar que los sistemas de información son un conjunto de componentes relacionados que recolectan (o recuperan), procesan, almacenan y distribuyen información para apoyar la toma de decisiones y el control en una organización. (Instituto Tecnológico de Sonora, 2014). Además de facilitar el archivo y la organización de cualquier tipo de información de forma rápida y sencilla, situación que optimizará las redes de mercadeo de los mercados tungurahuenses.

\section{Materiales y métodos.}

Para la realización del presente proyecto de investigación fue necesario la utilización de los métodos de investigación más apropiados basado en el autor (Woodsong, 2011, pág. 7) quien expone que "La investigación y el método científico proporcionan al profesional en su respectiva disciplina una perspectiva de análisis crítico de la información que maneja y de los conocimientos en los cuales fundamenta su acción profesional”, por otro lado en esta investigación fue necesario hacer uso del método inductivo, el cual "está generalmente asociado con la investigación cualitativa, ya que esta evita la cuantificación, recurriendo a hacer registros narrativos de los fenómenos que son estudiados mediante técnicas como la observación participante, las encuestas y entrevistas no estructuradas" (Díaz, 2014, pág. 28) por otro lado se utilizó la investigación de campo o investigación directa "es la que se efectúa en el lugar y tiempo en que ocurren los fenómenos objeto de estudio" (Grajales, 2015) por lo tanto en esta investigación se realizó un levantamiento de información en los mercados de la provincia de Tungurahua específicamente a los administradores de los mismos por medio de la aplicación práctica de las encuestas y se tabuló y analizó los resultados de la misma, comprobando de este modo los diferentes criterios de cada uno de los entrevistados, "lo cual permitió obtener conclusiones generales a partir de estos antecedentes particulares, 
además por medio de este método científico, se pudo distinguir cuatro pasos esenciales como la observación de los hechos para su registro; la clasificación de estos hechos; lo que permite llegar a una generalización; y la contrastación, tras una primera etapa de observación, análisis y clasificación de los hechos, se logra postular una posible solución al problema planteado". (Carazo, 2015). Es el conjunto total de individuos, objetos o medidas que poseen algunas características comunes observables en un lugar y en un momento determinado. Cuando se vaya a llevar a cabo alguna investigación debe de tenerse en cuenta algunas características esenciales al seleccionarse la población bajo estudio. (Wigodski, 2014); por lo tanto la población de esta investigación se establece en la cantidad de administradores de la red de mercados de Tungurahua mismos que ascienden a 17 personas; En vista de que la población de estudio es inferior a 100 se procederá a trabajar con el total de la población, de tal manera que no es necesario determinar la muestra de trabajo, en vista que la población o universo es muy pequeño, puesto que según el autor (Silva, 2013) menciona que "Si la población es pequeña y se puede acceder a ella sin restricciones, entonces se trabajará con toda la población. Si la población es muy grande o es demasiado costoso trabajar con toda la población, entonces conviene utilizar una muestra"

\section{Tabla 1.Detalle de la población}

\begin{tabular}{|c|c|c|c|c|}
\hline \multicolumn{5}{|c|}{ Mercados de Ambato con sus administradores } \\
\hline No. & Plaza o mercado & Administrador & Teléfono & \\
\hline 1.- & Plaza primero de mayo & Sr. Luis ramón & $\begin{array}{l}2997800 \\
7895\end{array}$ & ext. \\
\hline 2.- & Plaza pachano & Sr. Luis ramón & $\begin{array}{l}2997800 \\
7895\end{array}$ & ext. \\
\hline 3.- & Plaza sta. Clara & Sr. Geovanny sánchez & $\begin{array}{l}2997800 \\
7895\end{array}$ & ext. \\
\hline 4.- & Plaza juan cajas & Sr. Luis valle & $\begin{array}{l}2997800 \\
7895\end{array}$ & ext. \\
\hline 5.- & Cubículos control norte & Sr. Geovanny sánchez & $\begin{array}{l}2997800 \\
7895\end{array}$ & ext. \\
\hline 6.- & Cubículos huachi el belé & Sr. Geovanny sánchez & $\begin{array}{l}2997800 \\
7895\end{array}$ & ext. \\
\hline 7.- & C.c. Ferroviario & Sr. Darwin bolaños & $\begin{array}{l}2997800 \\
7895\end{array}$ & ext. \\
\hline 8.- & Mercado la dolorosa & Tlgo. Edwin fierro & $\begin{array}{l}2997800 \\
7895\end{array}$ & ext. \\
\hline 9.- & Mercado sur & Tlgo. Edwin fierro & $\begin{array}{l}2997800 \\
7895\end{array}$ & ext. \\
\hline 10.- & Mercado urbina y cubículos & Ing. Andrés crespo & $\begin{array}{l}2997800 \\
8717\end{array}$ & ext. \\
\hline 11.- & Mercado central y c.c de las flores & Ing. Javier jácome & $\begin{array}{l}2997800 \\
8725\end{array}$ & ext. \\
\hline 12.- & C.c. Artesanal & Ing. Miguel ávila & $\begin{array}{l}2997800 \\
8722\end{array}$ & ext. \\
\hline 13.- & Mercado modelo & Lic. Pablo sánchez & $\begin{array}{l}2997800 \\
8724\end{array}$ & ext. \\
\hline 14.- & C.c. Popular simón bolívar & Ing. Fernando arias & $\begin{array}{l}2997800 \\
8717\end{array}$ & ext. \\
\hline 15.- & Mercado américa & Ing. Ignacio carrasco & 408083 & \\
\hline
\end{tabular}


total

17 personas

\section{Fuente: (GAD MUNICIPALIDAD DE AMBATO, 2016)}

Una vez determinada la muestra de estudio se establece las técnicas e instrumentos de recolección por lo que se utilizó la técnica de la encuesta, misma que fue aplicada a la población de estudio, para luego proceder a tabular los resultados obtenidos por medio del programa IBM SPSS 23, además del apoyo de la hoja de cálculo Excel, y de esta manera se realizó un análisis e interpretación de los resultados obtenidos mismo que según el autor (Pérez, 2014, pág. 6) "el análisis de resultados consistirá en explicar los resultados obtenidos y comparar estos con datos obtenidos por otros investigadores, es una evaluación crítica de los resultados desde la perspectiva del autor tomando en cuenta los trabajos de otros investigadores y el propio". Siguiendo con la investigación se realizó la operacionalización de las variables por medio de las cuales se pudo determinar con claridad las preguntas apropiadas para la encuesta asi lo afirma el autor (Alvarez, 2016) que dice que "La operacionalización de variables es el proceso de transformación de una variable en otras que sean susceptibles de medir. Para lograr esto se descomponen en otras más específicas llamadas dimensiones y éstas se transforman en indicadores y por medio de estos se generan los ítems o preguntas para la encuesta". Luego se procedió a realizar la validación del método de recolección es decir de la encuesta y su contenido por medio del método de validación de Alfa de Cronbach, ya que La validez de un instrumento se refiere al grado en que el instrumento mide aquello que pretende medir. Y la fiabilidad de la consistencia interna del instrumento se puede estimar con el alfa de Cronbach. La medida de la fiabilidad mediante el alfa de Cronbach asume que los ítems (medidos en escala tipo Likert) miden un mismo constructo y que están altamente correlacionados (Welch \& Comer, 2015). Cuanto más cerca se encuentre el valor del alfa a 1 mayor es la consistencia interna de los ítems analizados. La fiabilidad de la escala debe obtenerse siempre con los datos de cada muestra para garantizar la medida fiable del constructo en la muestra concreta de investigación. (Frias, 2016), como se puede ver a continuación:

Dónde:

$$
\alpha=\frac{K}{K-1} 1-\frac{V_{i}}{V_{t}}
$$

$\alpha$ Alfa de Cronbach (entre 0 y 1 ).

$K$ Número de ítems (15).

$\sum \mathrm{V}_{\mathrm{i}}$ Sumatoria de las varianzas muéstrales de cada ítem $=14,55$

$V_{t}$ Varianza de las calificaciones individuales de la encuesta $=39.76$

$$
\begin{gathered}
\alpha=\frac{15}{15-1} 1-\frac{14.55}{18.49} \\
\alpha=\frac{15}{14} \quad 0.7869
\end{gathered}
$$




$$
\alpha=0.843
$$

Calculo que se lo realizo también con el programa IBM SPSS 23 obteniendo los mismos resultados como se puede apreciar

Tabla 2. Cálculo de Alfa de Cronbach.

\begin{tabular}{|ll|l|l|}
\hline & & $\mathrm{N}$ & $\%$ \\
\hline Casos & Válido & 17 & $100,0 \%$ \\
& Excluido $^{\mathrm{a}}$ & 0 &, $0 \%$ \\
& Total & 17 & $100,0 \%$ \\
\hline
\end{tabular}

La eliminación por lista se basa en variables del procedimiento.

\begin{tabular}{|l|l|}
\hline $\begin{array}{l}\text { Alfa de de } \\
\text { Cronbach }\end{array}$ & $\begin{array}{l}\text { N } \\
\text { elementos }\end{array}$ \\
\hline, 843 & 15 \\
\hline
\end{tabular}

\section{Fuente: IBM SPSS 23}

En la tabla de fiabilidad se puede determinar la confiabilidad del resumen del instrumento de medición como es la encuesta la misma que demuestra la totalidad de los casos presentados que en este caso son 17 que representa la totalidad de empresas encuestadas en el sector de agroalimentos que aceptaron a la prueba piloto inicial, así como el nivel de exclusión fue nula, debido a que el 100\% de las empresas encuestadas dieron su aceptación al proceso de investigación. En la tabla de resumen estadístico de fiabilidad presenta como resultado del .843 dando una calificación altamente confiable garantizando la validez de la investigación.

\section{Resultados.}

En base a la realización de este proyecto de investigación, se logrará aportar al sector de agroalimentos de Tungurahua ya que se optimizarán los procesos comerciales individuales de los comerciantes y productores, por medio de la implementación de un sistema de información para la red de plazas y mercados de Tungurahua, esta afirmación se basa en los resultados de la encuesta aplicada, donde se obtuvo los siguientes resultados por parte de los encuestados.

\section{Análisis.}

De acuerdo a los resultados se puede ver que el 70,6\% de los encuestados dicen que un sistema de información si sería la solución más apropiada para mantener informados a los productores y comerciantes acerca de los temas trascendentales para su negocio y para la comercialización de agroalimentos debido a que la red de plazas y mercados de Tungurahua nunca cumple a cabalidad con brindar la información necesaria acerca de los factores 
influyentes que intervienen en el sector de agroalimentos, demostrando de esta manera la gran necesidad de la implementación de un sistema de información, y certificando que el estudio es completamente viable, como se muestra a continuación.

Tabla 3. Solución de información

\begin{tabular}{ccccc}
\hline Válido & Frecuencia & Porcentaje & $\begin{array}{c}\text { Porcentaje } \\
\text { válido }\end{array}$ & $\begin{array}{c}\text { Porcentaje } \\
\text { acumulado }\end{array}$ \\
\hline SI & 12 & $70,60 \%$ & $70,60 \%$ & $70,60 \%$ \\
NO & 5 & $29,40 \%$ & $29,40 \%$ & $100,00 \%$ \\
Total & 17 & $100,00 \%$ & $100,00 \%$ & \\
\hline
\end{tabular}

Fuente: Investigación de campo (Encuesta)

Análisis:

Por otro lado se puede ver también la importancia que los encuestados le dan a la implementación de un sistema de información según se puede ver en la investigación donde se pregunta a los encuestados que en una escala del 1 al 5 donde: 1 es nada importante y 5 en muy importante ¿cómo califica la implementación de un sistema de información para la red de plazas y mercados? Donde el 70,6\% que corresponde a 12 personas dicen que es muy importante puesto que "Estos sistemas de información integrados son muy importantes para la organización, puesto que asegura que la información se comparta con todos los usuarios quienes tengan autorizado usarlo" (Zevallos, Pastor, \& Moscoso, 2016) por medio del cual se obtuvo los siguientes resultados que se pueden apreciar en el siguiente cuadro.

Tabla 4. Aporte al sector de agroalimentos.

\begin{tabular}{lcccc}
\hline \multicolumn{1}{c}{ Válido } & Frecuencia & Porcentaje & Porcentaje válido & Porcentaje acumulado \\
\hline POCO IMPORTANTE & 1 & $5,90 \%$ & $5,90 \%$ & $5,90 \%$ \\
IRRELEBANTE & 1 & $5,90 \%$ & $5,90 \%$ & $11,80 \%$ \\
IMPORTANTE & 3 & $17,60 \%$ & $17,60 \%$ & $29,40 \%$ \\
MUY IMPORTANTE & 12 & $70,60 \%$ & $70,60 \%$ & $100,00 \%$ \\
Total & $\mathbf{1 7}$ & $\mathbf{1 0 0 , 0 0 \%}$ & $\mathbf{1 0 0 , 0 0 \%}$ & \\
\hline
\end{tabular}

Fuente: (Zevallos, L. ; Pastor, R. ; Moscoso, B. 2011)

No hay que olvidar que se necesita estar completamente informados sobre todos los pormenores del sector agrícola, la entrada de contrabando de productos agroalimentarios, los costos de los insumos agrícolas, las fluctuaciones de precios debido a la especulación de los intermediarios etc. Situaciones que pueden ejercer modificaciones de los costos de venta por lo tanto los sistemas de información mantendrán informados a los comerciantes y productores acerca de estos menesteres, para así poder competir ya que en lo que tiene que ver con la oferta y la demanda de este tipo de productos está determinado por los costos es 
por eso que el método clásico para estimar los mismos está basado en la información que los comerciantes reciban, la cual a pesar de que implica ciertos costos y tiene márgenes de error es la más adecuada (Rosselli, D; Otero, A. ; et al, 2001).

Para diseñar un sistema de información para el sector de agroalimentos se procedió a hacer un análisis de del sector a nivel tecnológico por medio de un estudio de mercado en la provincia de Tungurahua donde se pudo encontrar las necesidades de información en el mercado y el movimiento de productos a los diferentes lugares de acopio, el desarrollo de la información y la rapidez con la que se transmita mejorará sustancialmente la movilidad de los mismos, además con la implementación de los sistemas de información se logrará tener eficiencia tanto en recursos económicos, como en ahorro de tiempo, logrando una ventaja competitiva en el mercado.

Gráfico 1. Uso de las TIC en el Ecuador.

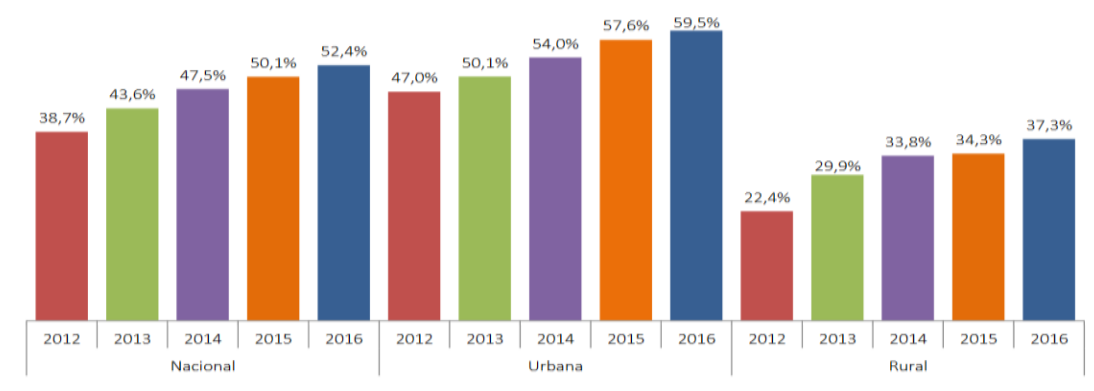

Fuente: (INEC, 2017)

El crecimiento del uso de las TIC a nivel nacional se ha incrementado a partir del año 2012 el cual muestra una tendencia a incrementar, logrando que en el 2016 más del 50\% de la población, tenga acceso a las TICS, se debe considerar que en las zonas urbanas donde el comercio tiene mayor afluencia, se muestra que un 59\% de la población conoce del uso de las TICS, lo cual se convierte en una ventaja, porque en el medio empresarial para lograr un mejor apalancamiento se optan por promocionar sus productos y servicios a través de medios informáticos. El incremento de los sistemas de información, permitirá crear un diseño de información para el sector de agroalimentos en la provincia de Tungurahua, lo que será de mayor accesibilidad para los usuarios del mercado dando facilidades de tiempo y recursos en la información requerida en el mercado. (Ministerio de Telecomunicaciones, 2016).

Por otro lado se estableció cual es nivel jerárquico de los sistemas de información El nivel estratégico está integrado por los directores los cuales tienen a cargo la toma de decisiones en base a la información del sistema de información, la misma que permitirá realizar análisis de los datos obtenidos en busca de resultados que permitan el crecimiento empresarial. El área administrativa estará dirigida por el gerente o administrador titular, el mismo que pondrá en ejecución las decisiones tomadas por los directores y verificara las actividades de trabajo bajo el sistema de información implementado por la empresa, permitiendo optimizar 
recursos. Los trabajadores de los sistemas de información que ocupan el nivel del conocimiento analizarán los datos obtenidos para su verificación permitiendo despejar las necesidades y demandas del mercado en el menor tiempo logrando cumplir los objetivos plantados en la satisfacción de necesidades y cumplimiento de objetivos. El nivel operativo verificará los sistemas de información para los registros de los procesos de las actividades diarias realizadas por la empresa los mismos que estarán en constante comunicación con los distintos niveles para aplicar las tareas establecidas en el proceso continuo de los sistemas de información conformando una cadena de información y trabajo constante dentro de la empresa.

Gráfico 2. Nivel sistemas de información.

\section{Nivel Jerárquico}

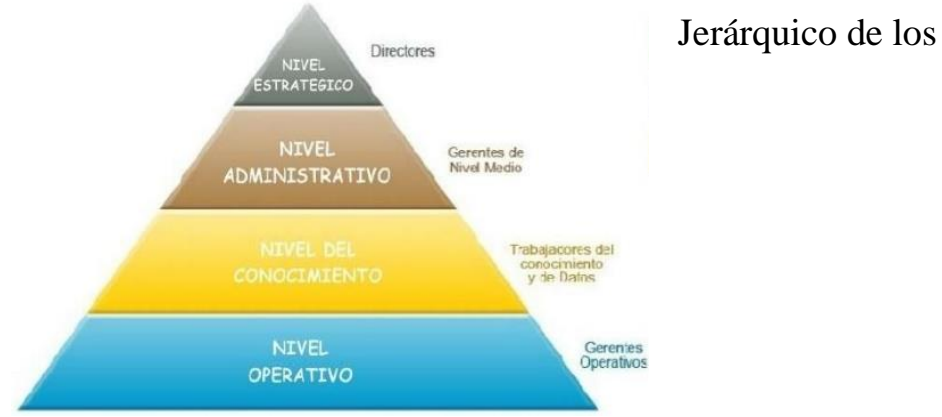

Fuente: ( Guayllaguaman , 2015)

Continuando con la investigación se procedió a definir la estructura de la base de datos de un sistema ya que para la implementación de los sistemas de información el requerimiento principal es la búsqueda de información veraz, que será ingresada en el sistema para ser analizada y catalogada en base a las necesidades del sistema a implementarse, esta información de datos deberá ser confiable con la finalidad de obtener los resultados esperados. El diseño conceptual está basado en una fase delicada porque precede inmediatamente aquella muy importante del análisis del sistema, para la cual los objetivos y justificaciones deben haber sido ya definidos dentro de la empresa, en cuanto al diseño lógico se realizara un esquema de información que utilizará la empresa basándose en datos independientes y específicos que le permitan analizar la información de manera oportuna y sistemática. (Marlats, Denegri, \& Lanfranco, 2015) 
Gráfico 3. Etapas para el diseño de una base de datos.

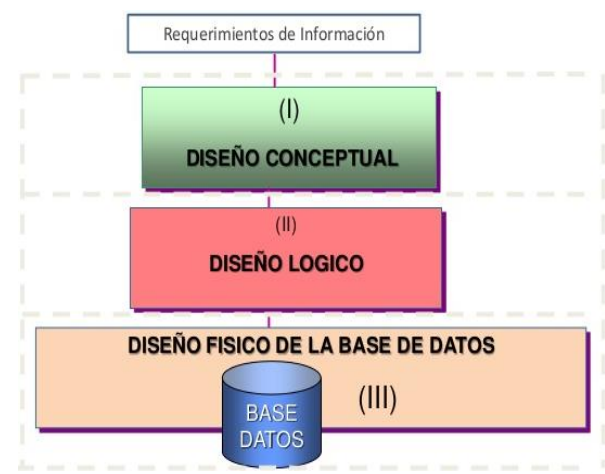

Fuente: (Sanchez, 2013)

Una vez determinadas las jerarquías y la estructura de los sistemas de información se procedió a determinar o seleccionar la herramienta tecnológica apropiada para su uso en la red de plazas y mercados de Tungurahua, por lo que para manejar la información del sector de agroalimentos de los mercados de la provincia de Tungurahua fue necesario la utilización del software "Alvendi Pro" Sistema para la gestión de información, almacenamiento, clasificación y organización de información, control de inventarios en cuanto a ventas, compras, cobros a clientes o pago a proveedores. (Laundon, 2015); De la misma manera se definió las fases de descubrimiento de la información en las cuales se pudo determinar las siguientes:

- Obtención de datos

- Recopilación

- Selección, ingreso y transformación

- Modelos a seguir

- Interpretación y evaluación (Bravo, 2017)

Luego de haber establecido todos los parámetros relevantes para esta investigación se procede a proponer como alternativa de solución la implementación de un diseño de sistema de información de mercado para el sector de agroalimentos de la Provincia de Tungurahua, específicamente en el departamento de la Red de Plazas y Mercados de la provincia de Tungurahua la cual será la encargada del manejo del sistema y de la información que será socializada a los diferentes mercados de la provincia, la red de plazas y mercados es una institución ecuatoriana ubicada en la provincia de Tungurahua cantón Ambato, la misma que desarrolla sus actividades a nivel local en donde el departamento de red de plazas y mercados apoya al incremento de sistemas de información para su mayor control y participación en el mercado. (Llanusa, Rojo, Caraballoso, Roberto, \& Pérez, 2015) 
Gráfico 4. Logotipo de red de plazas y mercados.

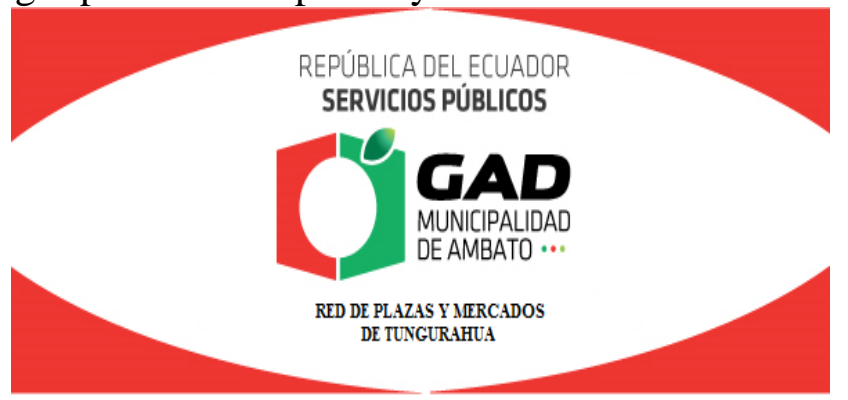

Fuente: Municipio de Ambato.

El sistema de información de la red de plazas y mercados serán identificados por medio del logotipo puesto que "a partir de la creación de un logotipo se puede generar un vínculo entre la empresa y sus públicos pues esta es una forma de plasmar una garantía sobre el servicio o productos, las primeras relaciones entre la imagen empresarial y el público, se materializaban a través de importantes vínculos de confianza. (Correa \& Orozco, 2015). Luego de este proceso se continúa determinando el organigrama estructural de la empresa específicamente del área donde se aplicó el sistema de información es decir en el departamento del área de servicios donde se encuentra la oficina de la red de plazas y mercados de la provincia de Tungurahua, la estructura organizativa se expone a continuación.

Gráfico 5. Organigrama del área de servicios.

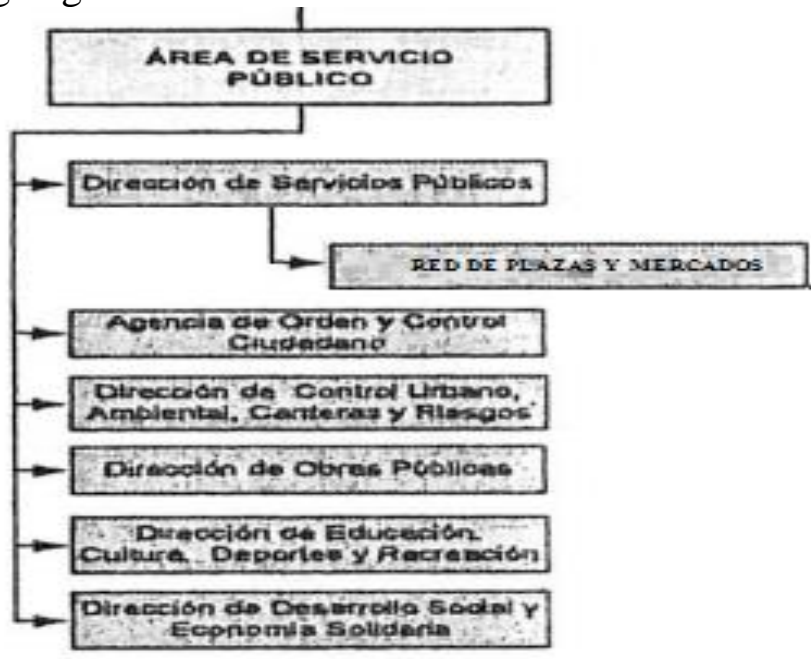

Fuente: (GAD MUNICIPALIDAD DE AMBATO, 2016)

Determinado el organigrama se procedió a realizar un análisis de los perfiles más importantes a los cuales se dará seguimiento por medio del sistema y para obtener la información oportunamente y llevar el control de los mismos (Yrusta \& Nardi, 2015); para mantener los procesos en orden en beneficio de los comerciantes y productores del sector de agroalimentos, perfiles que se detallan a continuación:

\section{Perfil financiero.}

Gráfico 6. Perfil Financiero. 


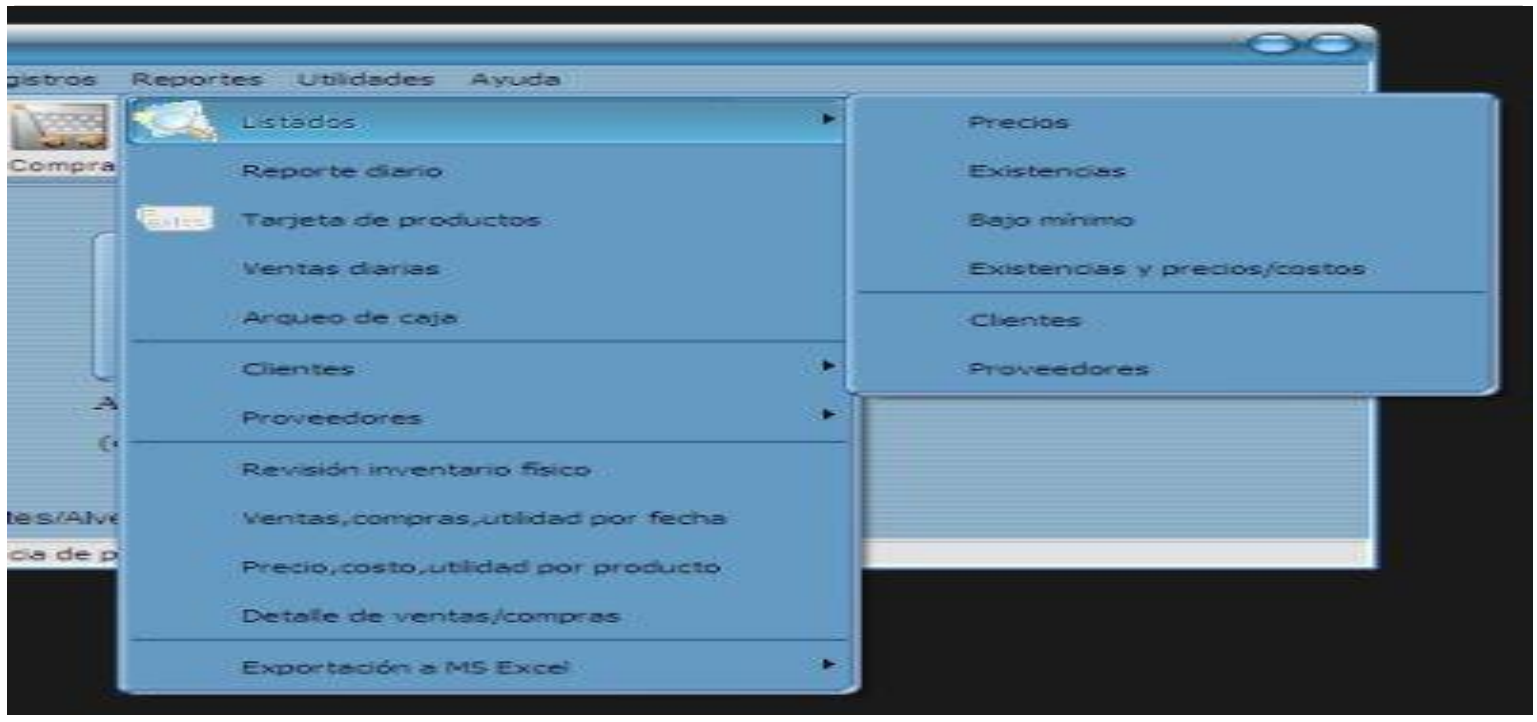

Fuente: (Plaza, Núquesi, \& Mejía, 2016)

Perfil de tecnologías de información.

Gráfico 7. Perfil de tecnologías de la información.

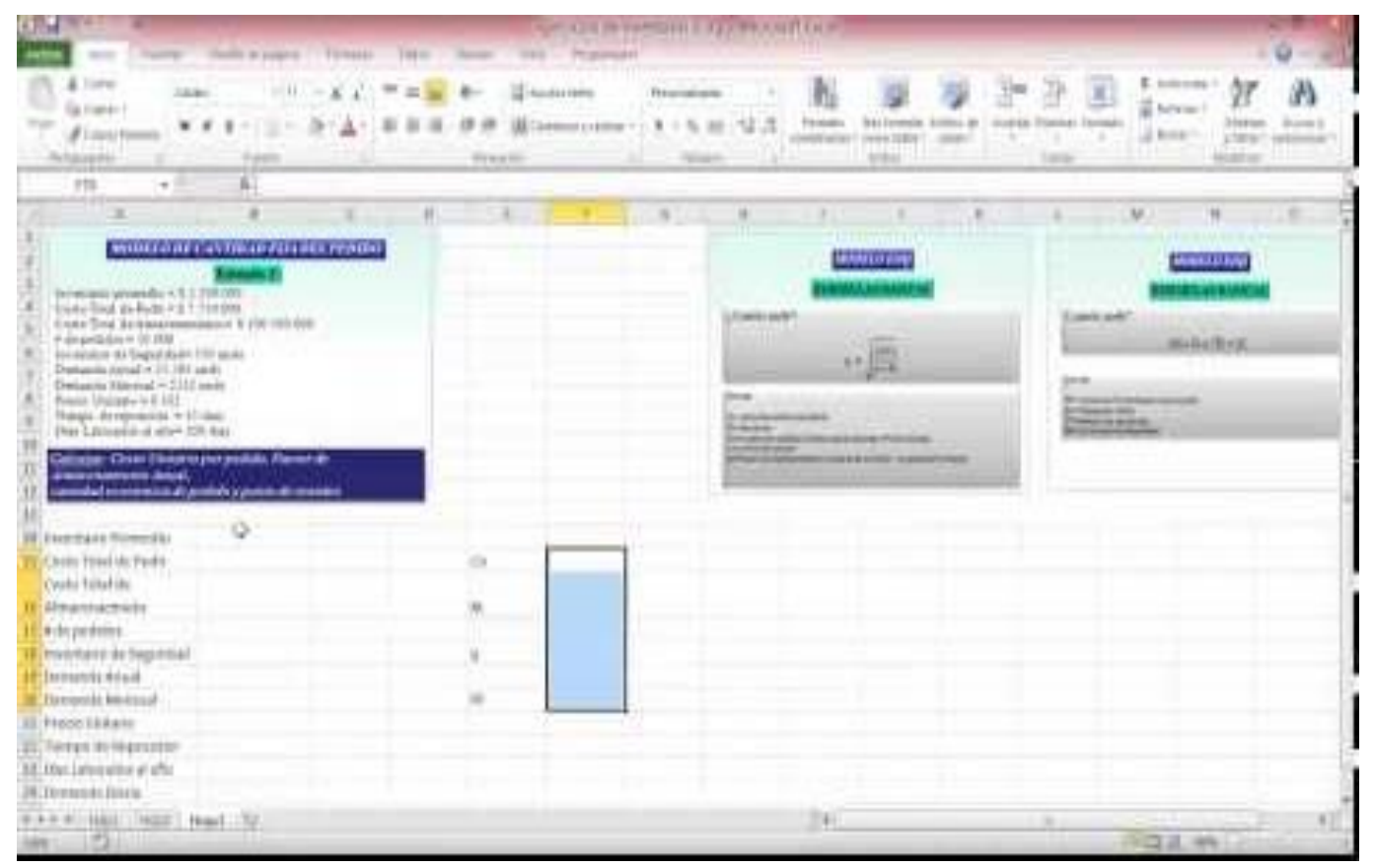

Fuente: (Yrusta \& Nardi, 2015)

\section{Perfil de la Marketing.}


Gráfico 8. Perfil de marketing.

Sistema de Irfiomación

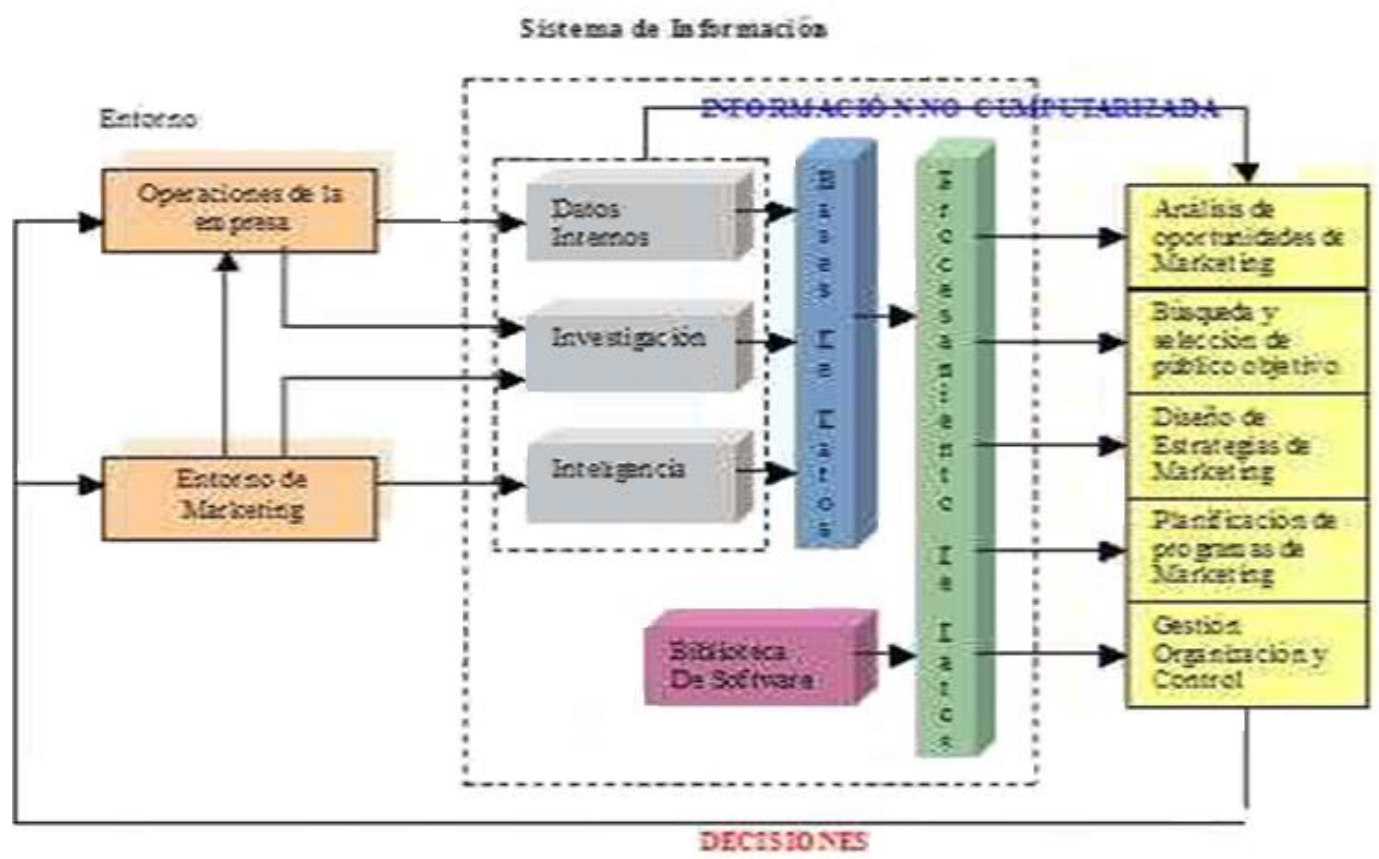

Fuente: (Marlats, Denegri, \& Lanfranco, 2015)

\section{Perfil de Ventas.}

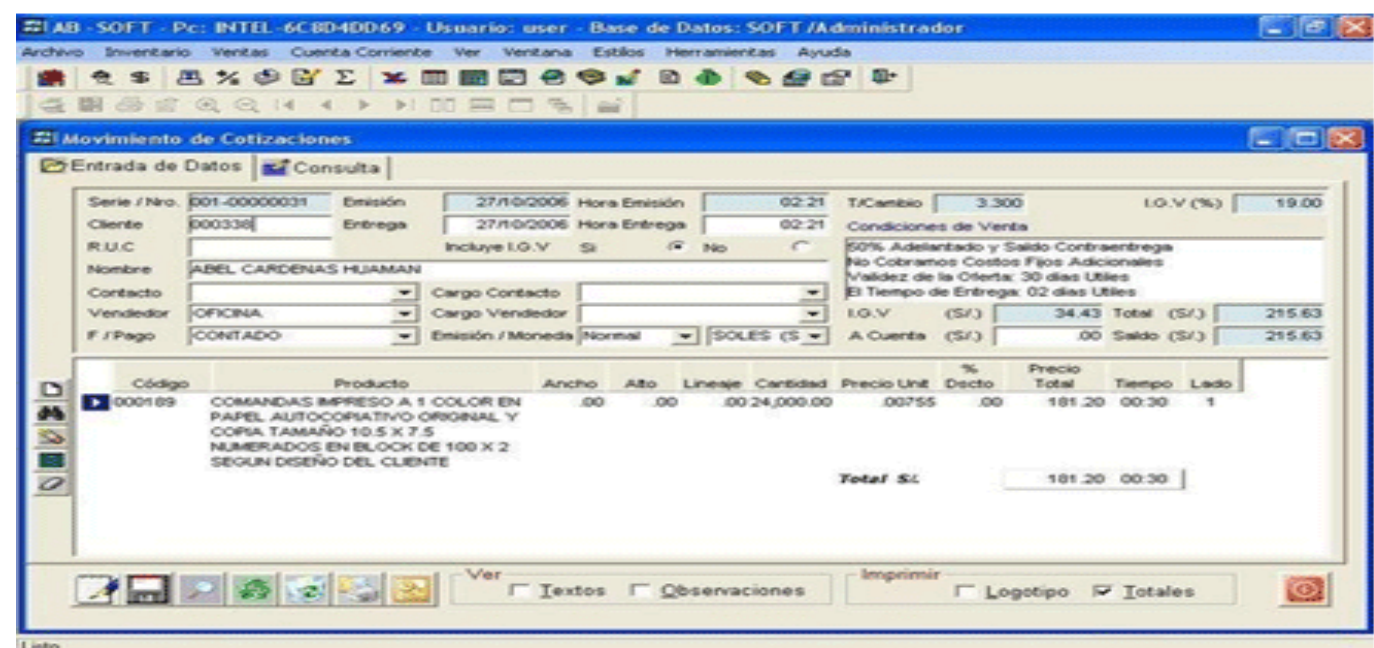

Fuente: (Taquía, 2016)

\section{Discusión.}

La aplicación del sistema de información para el sector de agroalimentos se la llevo a cabo en base a que el 70,6\% de los encuestados están completamente de acuerdo en que el sistema de información propuesto sería la solución más viable al problema de estudio, puesto que el mismo generará factores beneficiosos para el sector en discusión, en vista de que sus 
herramientas permitirán optimizar los procesos de comercialización de los comerciantes y productores de agroalimentos, además de generar un desarrollo sostenido del sector agrícola, no hay que dejar de lado el que la mayoría de los encuestados que alcanza a un 70,6\% que corresponden a una cantidad de 12 personas, mismas que desempeñan el cargo de administradores de los mercados en investigación, manifiestan por medio de la investigación que dichos sistemas de información son muy importantes para encaminar a los comerciantes y productores a tomar decisiones acertadas en cuanto a los factores que involucran a la comercialización de sus productos, beneficiando de esta manera y de forma directa a la ciudadanía en general ya que podrán adquirir los productos a precios reales y sin intermediarios lo cual dinamizará el sector económico de la provincia de Tungurahua, además de optimizar el área laboral del sector ya que a mayor ventas mayor producción lo cual será un pilar fundamental para fomentar el trabajo o para incrementar el sector laboral beneficiando sobre manera a los diferentes sectores que de la provincia que se dedican al cultivo agrícola, aportando de esta forma al mejoramiento de su estabilidad económica y por ende a su estilo de vida, lo cual generará el bienestar de muchas familias de los sectores agrícolas de la provincia, por lo tanto este proyecto debe ser aplicado con la mayor urgencia, con el compromiso de mantenerlo vigente y activo para sus usuarios en todo momento y llevando el control apropiado para evitar que se presenten inconvenientes que puedan afectar tanto a los comerciantes y productores, como al sector de agroalimentos de la provincia de Tungurahua.

\section{Conclusiones.}

Al haber realizado los estudios pertinentes para solventar la factibilidad y viabilidad de este proyecto de investigación, se ha llegado a las siguientes conclusiones.

- Pronosticar las diferentes situaciones que podrían afectar a la comercialización de un producto implica la estimación anticipada del valor de una variable (Pérez, I. ; Cifuentes, A.; et al. 2013). Por lo tanto se concluye que es necesario manejar la información de manera apropiada y con anticipación para hacer uso de la misma en beneficio de los usuarios del sistema de información, mismos que son los entes generadores de la necesidad principal de este estudio.

- Los resultados o parámetros obtenidos en la investigación, los cuales en términos comerciales, son el punto de actividad comercial (volumen de ventas) en donde los ingresos son la fuente de subsistencia, es decir, es el punto de actividad en donde el modus vivendi está determinado por el volumen de ventas. (Fantonny, X. 2014) esto deja claro que el círculo de negocio de los comerciantes y productores del sector de agroalimentos pueden generar los ingresos necesarios para cubrir los costos de producción, y movilización de sus productos, todo esto en base al uso de la información recibida con anterioridad para lograr establecer precios competitivos de sus productos en el mercado y generando un mayor volumen de ventas.

- Se concluye que la implementación de este proyecto de investigación fue completamente acertado puesto que por medio del mismo se aporta al desarrollo económico de la Provincia de Tungurahua, ya que, el enfoque del desarrollo 
económico local se destaca fundamentalmente en las formas de producción no basadas tan sólo en la industria, sino en las características generales y locales del territorio (Alburquerque, F. 2004) específicamente el cantón Ambato, además de apoyar a la agricultura al consumir la materia prima propia de lugar y que se encuentra en el sector.

- Se concluye diciendo que los resultados obtenidos con este proyecto son completamente factibles, y se ha beneficiado notablemente a la sociedad en el aspecto nutricional, puesto que por medio de la comercialización de productos de calidad se ha logrado mejorar el estilo de vida de muchas personas y de la sociedad, ya que lo más resaltante de este proyecto es que el sistema de información será completamente eficiente y de fácil acceso se podrán alcanzar de esta manera los objetivos principales de esta investigación.(Cuya, S.; Cervantes, L. 2015) cumpliendo de esta manera con los parámetros del Buen Vivir, establecidos actualmente en el país.

\section{Referencias.}

Guayllaguaman , D. (22 de 09 de 2015). http://dorisaraceli.blogspot.com. Recuperado el 09 de 08 de 2017, de http://dorisaraceli.blogspot.com/2015/09/niveles-jerarquicos-deuna-empresa.html

Alvarez, R. (19 de Marzo de 2016). OPERACIONALIZACIÓN DE VARIABLES. METODOLOGIA DE LA INVESTIGACION. Obtenido de http://metinvc.blogspot.com/2012/02/t6a-variables-recurso-analitico.html

Bravo, A. (02 de 04 de 2017). timeofsoftware.com. Recuperado el 09 de 08 de 2017, de http://timeofsoftware.com/descubriendo-informacion/

Carazo, C. (21 de Agosto de 2015). El método de estudio de caso Estrategia metodológica de la investigación científica. Pensamiento y gestión, 1(20). Obtenido de http://rcientificas.uninorte.edu.co/index.php/pensamiento/article/viewFile/3576/230 1

Collazos, C. (22 de Junio de 2016). ALIMENTACIÓN Y NUTRICIÓN. Cien años de Cooperación al Perú, 1-13. Obtenido de http://www.paho.org/per/images/stories/PER/Documentos/cooperacion\%20peru/P AG05.pdf

Correa, D., \& Orozco, J. (22 de Noviembre de 2015). Construcción de imagen de marca y reputación a través de campanas publicitarias de RSC. Redalic Sphera Pública(11), 273-289. Obtenido de http://www.redalyc.org/pdf/297/29729580016.pdf

Díaz, P. (22 de Abril de 2014). Investigación cuantitativa y cualitativa . Unidad de Epidemiología Clínica y Bioestadística., 76-78. Obtenido de http://www.fisterra.com/mbe/investiga/cuanti_cuali/cuanti_cuali2.pdf

Frias, N. (22 de Enero de 2016). Alfa de Cronbach y consistencia interna de los ítems de un instrumento de medida. Estadistica moderna, 4. Obtenido de http://www.uv.es/ friasnav/AlfaCronbach.pdf 
GAD MUNICIPALIDAD DE AMBATO. (27 de Agosto de 2016). Acerca de nosotros: gadmatic.ambato.gob.ec. Obtenido de gadmatic.ambato.gob.ec: http://gadmatic.ambato.gob.ec/pcfiles/Proceso\%20Rendicion\%20de\%20Cuentas\% 202014/11.\%20\%20CUMPLIMIENTO\%20DE\%20LA\%20EJECUCI\%C3\%93N\% 20PRESUPUESTARIA/11.3.FUNCI\%C3\%93N\%20A)\%20\%E2\%80\%93\%20(HO JA\%2052).pdf

García, O., Gonzalez, H., Rivera, L., \& Rivas, E. (19 de Febrero de 2015). SISTEMAS DE INFORMACION GERENCIAL. UNIVERSIDAD DON BOSCO, 2-33. Obtenido de https://s3.amazonaws.com/academia.edu.documents/36710601/PORTAFOLIO_SI G.pdf?AWSAccessKeyId=AKIAIWOWYYGZ2Y53UL3A\&Expires $=1504644563$ $\&$ Signature $=$ YpAdlNrzjtRoSU\%2F8YPnGZKtnF\%2Bg\%3D\&response-contentdisposition=inline \%3B\%20filename\%3DPORTAFOLIO_SIG.pdf

Grajales, T. (08 de Marzo de 2015). TIPOS DE INVESTIGACION. Investipos. Obtenido de http://tgrajales.net/investipos.pdf

INEC. (09 de 08 de 2017). ecuadorencifras.gob.ec. Obtenido de http://www.ecuadorencifras.gob.ec/documentos/webinec/Estadisticas_Sociales/TIC/2016/170125.Presentacion_Tics_2016.pd

Instituto Tecnológico de Sonora. (1 de Abril de 2014). Acerca de nosotros: biblioteca.itson.mx. Obtenido de biblioteca.itson.mx Web site: http://biblioteca.itson.mx/oa/dip_ago/introduccion_sistemas/p3.htm

Laundon, k. P. (2015). Sistemas de información gerencial: administración de la empresa digital. Madrid. Obtenido de https://books.google.es/books?hl=es\&lr=\&id=KD8ZZ66PF$\mathrm{gC} \& \mathrm{oi}=\mathrm{fnd} \& \mathrm{pg}=\mathrm{PA} 210 \& \mathrm{dq}=\mathrm{que}+$ son+los+sistemas+de+informaci\%C3\%B3n+\&o ts $=$ hlhlubnZXD\&sig=Aur6Rw1G2rirqsJesfk4bqdN3vs\#v=onepage \&q=que $\% 20$ son \%201os\%20sistemas\%20de\%20informaci\%C3\%B3n\&f=false

Llanusa, S., Rojo, N., Caraballoso, M., Roberto, C., \& Pérez, J. (19 de Mayo de 2015). Las tecnologías de información y comunicación y la gestión del conocimiento en el sector salud. Revista Cubana de Salud Pública, 31(3), 24-95. Obtenido de http://scielo.sld.cu/scielo.php?script=sci_arttext\&pid=S0864-34662005000300008

Marlats, M., Denegri, G. A., \& Lanfranco, J. (29 de Junio de 2015). Sistemas de informacion silvopastoriles: estimación de beneficios directos comparados con monoculturas en la pampa ondulada, Argentina. CATIE(8), 186. Obtenido de http://repositorio.bibliotecaorton.catie.ac.cr/handle/11554/6827

Ministerio de Telecomunicaciones. (19 de Marzo de 2016). Plan Nacional de Telecomunicaciones y Tecnologias de la Información del Ecuador . 1-66. Obtenido de https://www.telecomunicaciones.gob.ec/wp-content/uploads/2016/08/Plan-deTelecomunicaciones-y-TI..pdf

Pérez, J. (08 de Mayo de 2014). CÓMO REDACTAR EL ANÁLISIS DE LOS RESULTADOS. Asesoria. Obtenido de http://asesoriatesis1960.blogspot.com/2010/12/analisis-de-los-resultados.html

Plaza, D., Núquesi, A., \& Mejía, M. (17 de Abril de 2016). Implementación del sisytema de negociacion bursatilpara las pimes del Ecuador. Revista Tecnologica ESPOL, 20(20), 
200.

Obtenido

https://www.dspace.espol.edu.ec/bitstream/123456789/8387/1/Implementaci\%C3\% B3n\%20de1\%20Sistema\%20de\%20Negociaci\%C3\%B3n\%20Burs\%C3\%A1til\%20 de $\% 20$ cheques.pdf

Sanchez, I. (7 de 12 de 2013). slideshare.net. Recuperado el 09 de 08 de 2017, de https://es.slideshare.net/mickienet/base-de-datos-sistema-modelo-de-gestion-dedatos

Silva, A. (25 de Mayo de 2013). Acerca de nosotros: allanucatse.files.wordpress.com. Obtenido de allanucatse.files.wordpress.com Web site: https://allanucatse.files.wordpress.com/2011/01/tipo-de-muestreo.pdf

Taquía, J. (22 de Mayo de 2016). Retail marketing para desarrollar mercados. Redalic(33), 133-155. Obtenido de http://www.redalyc.org/articulo.oa?id=337443854006

Welch, F., \& Comer, M. (2015). Alfa de Cronbach y consistencia interna de los ítems de un instrumento de medida. Estadistica Moderna, 22. Obtenido de http://www.uv.es/ friasnav/AlfaCronbach.pdf

Wigodski, J. (02 de Mayo de 2014). Acerca de nosotros: metodologiaeninvestigacion.blogspot.com. metodologiaeninvestigacion.blogspot.com Obtenido de http://metodologiaeninvestigacion blogspot com/2010/07/pobla

Woodsong, C. (16 de Julio de 2011). Qualitative Research Methods: A DATA COLLECTOR'S FIELD GUIDE. amily Healt International, 137. Obtenido de https://www.fhi360.org/sites/default/files/media/documents/Qualitative\%20Researc h\%20Methods\%20-\%20A\%20Data\%20Collector\%27s\%20Field\%20Guide.pdf

Yrusta, L., \& Nardi, A. (24 de Julio de 2015). Oficina de Conocimiento Abierto: un modelo para institucionalizar el acceso abierto en las universidades. El profesional de la información, 21(6), 69-163. Obtenido de http://www.elprofesionaldelainformacion.com/contenidos/2012/noviembre/11.html

Zevallos, L., Pastor, R., \& Moscoso, B. (17 de Julio de 2016). OFERTA Y DEMANDA DE SSISTEMAS DE INFORMACIÓN EN LOS ESTABLECIMIENTOS DE SALUD DEL MINISTERIO DE SALUD: BRECHAS A NIVEL NACIONAL, POR REGIONES Y TIPO DE ESPECIALIDAD. Peru Med, 28(2), 177-85. Obtenido de www.rpmesp.ins.gob.pe/index.php/rpmesp/article/download/482/483 


\section{Para citar el artículo indexado.}

Velasteguí E, Carrasco T. \& Chávez W. (2018). Sistema de información de mercado para el sector de agroalimentos de los mercados de la Provincia de Tungurahua. Revista electrónica Visionario Digital 1(1), 5-24. Recuperado desde: http://cienciadigital.org/revistacienciadigital2/index.php/VisionarioDigital/article/view/33/32

\section{【L Ciencia}

El artículo que se publica es de exclusiva responsabilidad de los autores y no necesariamente reflejan el pensamiento de la Revista Ciencia Digital.

El articulo queda en propiedad de la revista y, por tanto, su publicación parcial y/o total en otro medio tiene que ser autorizado por el director de la Revista Ciencia Digital.
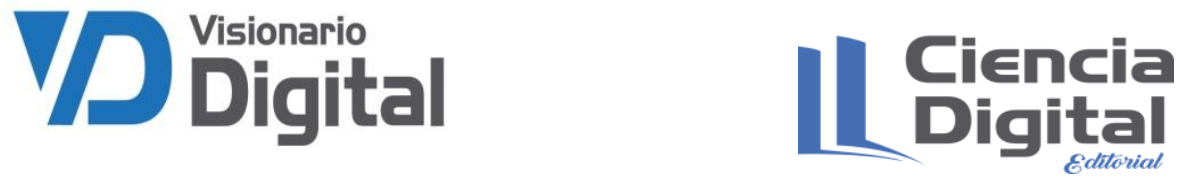